\title{
ON A CHARACTERIZATION OF LOCALLY COMPACT GROUPS OF SECOND CATEGORY, ASSUMING THE CONTINUUM HYPOTHESIS
}

\author{
INDER K. RANA
}

\begin{abstract}
Let $G$ be a topological group of second category and having cardinality at most that of the continuum. Let $B$ be some $\sigma$-algebra of subsets of $G$ such that $(G, B)$ is a measurable group. For a probability measure $P$ on $(G, B)$, write $P_{g}$ for the measure defined by $P_{g}(E)=P(g E)$, $E \in \mathbf{B}$. The aim of this paper is to prove the following: if on $(G, \mathbf{B})$ there exists an inner-regular probability measure $P$ such that $P_{g} \ll \mu$ for every $g \in G$, where $\mu$ is some $\sigma$-finite measure on $(G, \mathbf{B})$, then $G$ is locally compact. Further if $S$ denotes the $\sigma$-algebra generated by the topology of $G$ and $m$ denotes a Haar measure on $G$, then $\mu \gg m \gg P_{g}$ for every $g \in G$ on the $\sigma$-algebra $S \cap \mathbf{B}$.
\end{abstract}

1. Introduction. In [3] it was proved that given a standard measurable group $(G, \mathbf{B}), P$ a probability measure and $\mu$ some $\sigma$-finite measure on $(G, \mathbf{B})$ such that $P_{g} \ll \mu$ for every $g \in G$, where $P_{g}(E)=P(g E)$ for every $E \in \mathbf{B}$, there exists a topology $\tau$ on $G$ such that $(G, \tau)$ is a locally compact group, the $\sigma$-algebra generated by $\tau$ is $\mathbf{B}$ and if $m$ denotes the Haar measure of $(G, \tau)$, then $P_{g} \ll m \ll \mu$ for every $g \in G$. In the present paper we show, using the construction given in [3] and a result of [2], that topological groups of second category having cardinality at most that of the continuum (not necessarily standard) are locally compact if there exists an inner regular probability $P$ on some $\sigma$-algebra $\mathbf{B}$ of subsets of $G$ such that $(G, \mathbf{B})$ is a measurable group and $P_{g} \ll \mu$ for every $g \in G$, where $\mu$ is some $\sigma$-finite measure on $(G, \mathbf{B})$.

It may be remarked that we shall be assuming the continuum hypothesis.

2. Preliminaries. Let $X$ be a topological space and $\mu$ a measure defined on some $\sigma$-algebra $\mathbf{B}$ of subsets of $X . \mu$ will be called inner-regular if $\mu(E)=$ $\sup \{\mu(K): K \in \mathbf{B}, K \subset E$ and $K$ compact $\}$, for every $E \in \mathbf{B}$. $\mu$ will be called locally-finite if for every $x \in X$ there exists some neighbourhood $V_{x}$ of $x$ such that $\mu\left(V_{x}\right)<\infty$.

$G$ will stand for a topological group. $G$ is said to be of second category if the topology of $G$ is of second category. Let B be some $\sigma$-algebra of subsets of $G$. $\left(G\right.$, B) will be called a measurable group if the map $(g, h) \rightarrow g h^{-1}$ from $G \times G \rightarrow G$ is measurable when $G \times G$ is given the product $\sigma$-algebra. A measure $\mu$ on a measurable group $(G, \mathbf{B})$ is said to be (left) quasi-invariant if

Received by the editors July 6, 1976.

AMS (MOS) subject classifications (1970). Primary 43A05.

Key words and phrases. Measurable group, inner-regular measure, quasi-invariant measure.

(c) American Mathematical Society 1977 
$\mu(E)=0 \Leftrightarrow \mu(g E)=0$ for every $g \in G$. Here $g E=\{g h: h \in E\}$. By the translates of a measure $\mu$ on a measurable group $(G, \mathbf{B})$, denoted by $\left\{\mu_{g}\right\}$, we mean the measures $\mu_{g}$ defined by $\mu_{g}(E)=\mu(g E)$ for every $E \in \mathbf{B}$ and $g \in G$.

With the above notations we shall prove the following:

THEOREM. Let $G$ be a topological group of second category and having cardinality at most that of the continuum. If B is some $\sigma$-algebra of subsets of $G$ such that $(G, \mathbf{B})$ is a measurable group and if there exists an inner-regular probability measure $P$ on $\left(G\right.$, B) such that $P_{g} \ll \mu$ for every $g \in G, \mu$ being some $\sigma$-finite measure on $(G, \mathbf{B})$, then $G$ is locally compact. Moreover if $S$ denotes the $\sigma$-algebra generated by the topology of $G$ and $m$ denotes the Haar measure of $G$, then $\mu \gg m \gg P_{g}$ for every $g \in G$ on the $\sigma$-algebra $S \cap \mathbf{B}$.

Note that if $G$ is any locally compact group and if we take $\mathbf{B}=$ the natural Borel $\sigma$-algebra on $G$ and $P=\mu=$ some probability measure equivalent to the Haar measure of $G$, then all the conditions given in the theorem are satisfied.

For the proof of the theorem we need some elementary results. In the lemmas to follow $X$ will denote a topological space and B some $\sigma$-algebra of subsets of $X$.

LEMMA 1. (i) If $\mu$ is an inner-regular measure on $(X, \mathbf{B})$, then so is its restriction $\mu_{B}$ to any $B \in \mathbf{B}$, where $\mu_{B}(E)=\mu(B \cap E)$ for every $E \in \mathbf{B}$.

(ii) If $\left\{\mu_{n}\right\}$ is a sequence of inner-regular probability measures on $(X, \mathbf{B})$, then so is the measure $\nu$ defined by

$$
\nu(E)=\sum_{n=1}^{\infty} \frac{\mu_{n}(E)}{2^{n}} \quad \text { for every } E \in \mathbf{B}
$$

Proof. (i) Let $E \in \mathbf{B}$ and let $\varepsilon>0$ be given. Then by the inner regularity of $\mu$, there exists a compact set $K \subset B \cap E, K \in \mathbf{B}$, such that $\mu(B \cap E)-$ $\mu(K)<\varepsilon$. Since $K \subset E$ and $K \cap B=K$, we have

$$
\begin{aligned}
\mu_{B}(E)-\mu_{B}(K) & =\mu(B \cap E)-\mu(B \cap K) \\
& =\mu(B \cap E)-\mu(K)<\varepsilon,
\end{aligned}
$$

showing that $\mu_{B}$ is inner-regular.

(ii) Let $E \in \mathbf{B}$ and let $\varepsilon>0$ be given. Since $\sum_{n=1}^{\infty} \mu_{n}(X) / 2^{n}=1$, we can choose a positive integer $N$ such that $\sum_{n=N+1}^{\infty} \mu_{n}(X) / 2^{n}<\varepsilon / 2$. Now by inner-regularity of $\mu_{n}, n=1,2, \ldots, N$, there exist compact sets $K_{n} \in \mathbf{B}$, $K_{n} \subset E$, such that $\mu_{n}\left(E-K_{n}\right) / 2^{n}<\varepsilon / 2 \cdot N, n=1,2, \ldots, N$. Put $K=$ $\cup_{k=1}^{N} K_{n}$. Then $K$ is compact, $K \in \mathbf{B}$ and $K \subset E$. Moreover,

$$
\frac{\mu_{n}(E-K)}{2^{n}} \leqslant \frac{\mu_{n}\left(E-K_{n}\right)}{2^{n}}<\frac{\varepsilon}{2 \cdot N}, \quad n=1,2, \ldots, N .
$$

Then $\sum_{n=1}^{N} \mu_{n}(E-K) / 2^{n}<\varepsilon / 2$. Now 


$$
\begin{array}{r}
\nu(E-K)-\sum_{n=1}^{N} \frac{\mu_{n}(E-K)}{2^{n}}=\sum_{n=1}^{\infty} \frac{\mu_{n}(E-K)}{2^{n}}-\sum_{n=1}^{N} \frac{\mu_{n}(E-K)}{2^{n}} \\
=\sum_{n=N+1}^{\infty} \frac{\mu_{n}(E-K)}{2^{n}} \leqslant \sum_{n=N+1}^{\infty} \frac{\mu_{n}(K)}{2^{n}}<\frac{\varepsilon}{2} .
\end{array}
$$

Thus $\nu(E-K)<\sum_{n=1}^{N} \mu_{n}(E-K) / 2^{n}+\varepsilon / 2<\varepsilon / 2+\varepsilon / 2=\varepsilon$. Thus given $E \in \mathbf{B}$ and $\varepsilon>0$, we have found a compact set $K \in \mathbf{B}, K \subset E$, such that $\nu(E-K)<\varepsilon$, which proves that $\nu$ is inner-regular.

LEMMA 2. If $\mu$ and $\nu$ are two probability measures on $(X, \mathbf{B}), \nu$ inner-regular, then there exist measures $\nu_{1}$ and $\nu_{2}$, both inner-regular, such that $\nu=\nu_{1}+\nu_{2}$, $\nu_{1} \perp \mu$ and $\nu_{2} \ll \mu$.

Proof. Follows immediately from the proof of the well-known Lebesgue decomposition theorem (for example, the one given in [1]) keeping in view Lemma 1 . We will call $\nu=\nu_{1}+\nu_{2}$ the Lebesgue decomposition of the inner-regular measure $\nu$ with respect to $\mu$.

For the proof of the theorem, we will also be requiring the following result due to Kai-Wang $\mathrm{Ng}$, for a proof of which we refer to [2]. The result is as follows:

"Let $G$ be a topological group of second category. Then there exists a locally finite inner-regular, quasi-invariant measure on a $\sigma$-field $\mathbf{B}$ of subsets of $G$, such that $(G, \mathbf{B})$ is a measurable group, if and only if $G$ is locally compact."

3. Proof of the Theorem. We first prove that under the given hypothesis there exists a quasi-invariant inner-regular probability measure on $(G, \mathbf{B})$. To start with, note that since $P$ is inner-regular, so is $P_{g}$ for every $g \in G$. Further we can assume without any loss of generality that $\mu$ is a probability measure. In case $G$ is a countable group say $G=\left\{g_{i}\right\}$, it is obvious that

$$
\nu(E)=\sum_{i=1}^{\infty} \frac{P_{g_{i}}(E)}{2^{i}}, \quad E \in \mathbf{B},
$$

will be the required quasi-invariant, inner-regular probability measure. So let the cardinality of $G$ be that of the continuum. We choose a well-ordering ' $<$ ' of elements of $G$ such that for every $g \in G$, the set $\{h: h<g\}$ is countable (that such a well-ordering exists, having assumed the continuum hypothesis, we refer to p. 29 of General Topology by Kelley). Let the elements of $G$ be written as a transfinite sequence $g_{1}, g_{2}, \ldots, g, \ldots$ Put $P_{g_{1}}^{1}=P_{g_{1}}, P_{g_{1}}^{2}=0$. Let $P_{g_{2}}=P_{g_{2}}^{1}+P_{g_{2}}^{2}$ be the Lebesgue decomposition of $P_{g_{2}}$ with respect to $P_{g_{1}}^{1}$ (given by Lemma 2) where $P_{g_{2}}^{1} \perp P_{g_{1}}^{1}, P_{g_{2}}^{2} \ll P_{g_{1}}^{1}$ and both $P_{g_{2}}^{1}, P_{g_{2}}^{2}$ are inner-regular. Let $P_{g_{3}}=P_{g_{3}}^{1}+P_{g_{3}}^{2}$ be the Lebesgue decomposition of $P_{g_{3}}$ with respect to $P_{g_{1}}^{1}+P_{g_{2}}^{1}$ (given by Lemma 2). In general suppose $P_{h}^{1}$ and $P_{h}^{2}$ have been defined for every $h<g$. Let $P_{g}=P_{g}^{1}+P_{g}^{2}$ be the Lebesgue decomposition of $P_{g}$ (given by Lemma 2) with respect to $\sum_{h<g} C_{h}^{g} P_{h}^{1}$ where for every $g$, 
$\left\{C_{h}^{g}\right\}_{h<g}$ is some sequence of positive numbers such that $\sum_{h<g} C_{h}^{g} P_{h}^{1}$ is a probability measure. Then by transfinite induction we get the families $\left\{P_{g}^{1}\right\}$ and $\left\{P_{g}^{2}\right\}$ with the following properties:

(i) $P_{g}=P_{g}^{1}+P_{g}^{2}$.

(ii) $P_{g}^{1} \perp \Sigma_{h<g} C_{h}^{g} P_{h}^{1}, P_{g}^{2} \ll \Sigma_{h<g} C_{h}^{g} P_{h}^{1}$.

(iii) $P_{g}^{1}$ and $P_{g}^{2}$ are inner-regular.

Consider the family $\left\{P_{g}^{1}\right\}$. Note that for $g \neq h, P_{g}^{1} \perp P_{h}^{1}$ and $P_{g}^{1} \ll \mu$ for every $g \in G$. Thus $\left\{P_{g}^{1}\right\}$ is a family of measures of cardinality that of the continuum, its members are mutually orthogonal and are dominated by $\mu$. Thus $\left\{P_{g}^{1}\right\}$ satisfies the conditions of Lemma 2 of [3] and hence is countable say $\left\{P_{g(i)}^{1}\right\}$. Define $\nu(E)=\sum_{i=1}^{\infty} C_{i} P_{g(i)}^{1}(E), E \in \mathbf{B}$ (where $C_{i}$ is some sequence of positive numbers such that $\nu$ is a probability measure). Then it is easy to see that $\nu$ is quasi-invariant and it follows from Lemma 1 that $\nu$ is inner-regular also.

Thus under the given hypothesis on $(G, \mathbf{B})$ we have constructed an inner-regular quasi-invariant probability measure $\nu$ on $(G, \mathbf{B})$. Now we apply the result of Kai-Wang $\mathrm{Ng}$, and get that $G$ is locally compact.

Let $S$ denote the $\sigma$-algebra generated by the open subsets of $G$. Then $S \cap \mathbf{B}$ is a $\sigma$-algebra and is invariant under the group operations. Let $m$ denote the Haar measure of $G$. Then $m$ and $\nu$ are two quasi-invariant measures on $(G, S \cap B)$ and thus they must be equivalent (see [4, Theorem 3.1.5]). Thus $m \equiv \nu \gg P_{g}$ on $S \cap \mathbf{B}$ for every $g \in G$. Obviously from the construction of $\nu$ it follows that $\mu \gg \nu$ and thus $\mu \gg m \gg P_{g}$ on $S \cap \mathbf{B}$ for every $g \in G$. This completes the proof of the theorem.

\section{REFERENCES}

1. P. R. Halmos, Measure theory, Van Nostrand, Princeton, N. J., 1950. MR 11, 504.

2. Kai Wang $\mathrm{Ng}$, Quasi-invariant measures on groups of second category, J. London Math. Soc. 7 (1973), 170-174.

3. I. K. Rana, On a characterization of standard measurable groups, Sankhya (to appear).

4. Xia-Dao Xing, Measure and integration theory on infinite-dimensional spaces. Abstract Harmonic analysis, Academic Press, New York, 1972. MR 46 \#9281.

Indian Statistical Institute, 7, S.J.S. Sansanwal Marg, New Delhi 110029, India 